\title{
An Organic Electronic Ion Pump to Regulate Intracellular Signaling at High Spatiotemporal Resolution
}

\author{
Daniel Simon, Edwin Jager, Klas Tybrandt, Larsson, Sinduh Kurup, \\ Agneta Richter-Dahlfors and Magnus Berggren
}

\section{Linköping University Post Print}

N.B.: When citing this work, cite the original article.

C2009 IEEE. Personal use of this material is permitted. However, permission to reprint/republish this material for advertising or promotional purposes or for creating new collective works for resale or redistribution to servers or lists, or to reuse any copyrighted component of this work in other works must be obtained from the IEEE.

Daniel Simon, Edwin Jager, Klas Tybrandt, Larsson, Sinduh Kurup, Agneta Richter-Dahlfors and Magnus Berggren, An Organic Electronic Ion Pump to Regulate Intracellular Signaling at High Spatiotemporal Resolution, 2009, Transducers 2009: The 15th international conference on Solid-State Sensor, Actuators and Microsystems, 1790-1793.

http://dx.doi.org/10.1109/SENSOR.2009.5285721

Postprint available at: Linköping University Electronic Press

http://urn.kb.se/resolve?urn=urn:nbn:se:liu:diva-53036 


\title{
AN ORGANIC ELECTRONIC ION PUMP TO REGULATE INTRACELLULAR SIGNALING AT HIGH SPATIOTEMPORAL RESOLUTION
}

\author{
Daniel T. Simon ${ }^{1,3}$, Edwin W. H. Jager ${ }^{1,3^{*}}$, Klas Tybrandt ${ }^{1,3}$, Karin C. Larsson ${ }^{2,3}$, \\ Sindhulakshmi Kurup ${ }^{2,3}$, Agneta Richter-Dahlfors ${ }^{2,3}$, and Magnus Berggren ${ }^{1,3}$ \\ ${ }^{1}$ Department of Science and Technology, Linköping University, SE-601 74 Norrköping, Sweden \\ ${ }^{2}$ Department of Neuroscience, Karolinska Institutet, SE-171 77 Stockholm, Sweden \\ ${ }^{3}$ Strategic Research Center for Organic Bioelectronics, Sweden
}

\begin{abstract}
Current technologies for cell stimulation suffer from a variety of drawbacks. Indeed, precise, localized, and minimally disruptive machine-to-cell interfacing is difficult to achieve. Here we present the organic electronic ion pump (OEIP), a polymer-based delivery system exhibiting high spatial, temporal, and dosage precision. Based on electrophoretic transport of positively charged species, the OEIP can deliver - with high precision - an array of biologically relevant substances without fluid flow, thus eliminating convective disturbance of the target system's environment. We discuss our results to date, including oscillatory delivery profiles and stimulation of neuronal cells in vitro, as well as our ongoing work.
\end{abstract}

\section{KEYWORDS}

Organic electronics, drug delivery, polymer, electrochemistry, electrophoresis

\section{INTRODUCTION}

Conjugated polymer systems, doped and un-doped, can exhibit high conductivity for both ions and electrons. In their oxidized state, high concentrations of holes are easily transported along the $\pi$-conjugated backbone. Meanwhile, an open, amorphous bulk structure, in conjunction with the chemical properties of the polymerdopant ion system, provides for fast ion conduction. We have chosen the conducting polymer system poly $(3,4-$ ethylenedioxythiophene) doped with the polyanion poly(styrenesulfonate) (PEDOT:PSS) [1] (Figure 1) to achieve "ion pump" delivery devices that exploit this combined ionic and electronic conduction. Upon biasing these organic electronic ion pumps (OEIPs) [2], electronic addressing signals are converted into exact delivery of ions and bio-substances at very high spatiotemporal resolution,
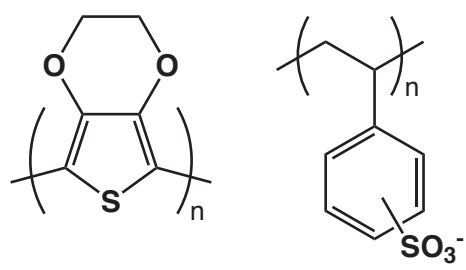

Figure 1: The $\pi$-conjugated conducting polymer poly(3,4ethylenedioxythiophene) (left) doped with the polyanion poly(styrenesulfonate) (right) (PEDOT:PSS).
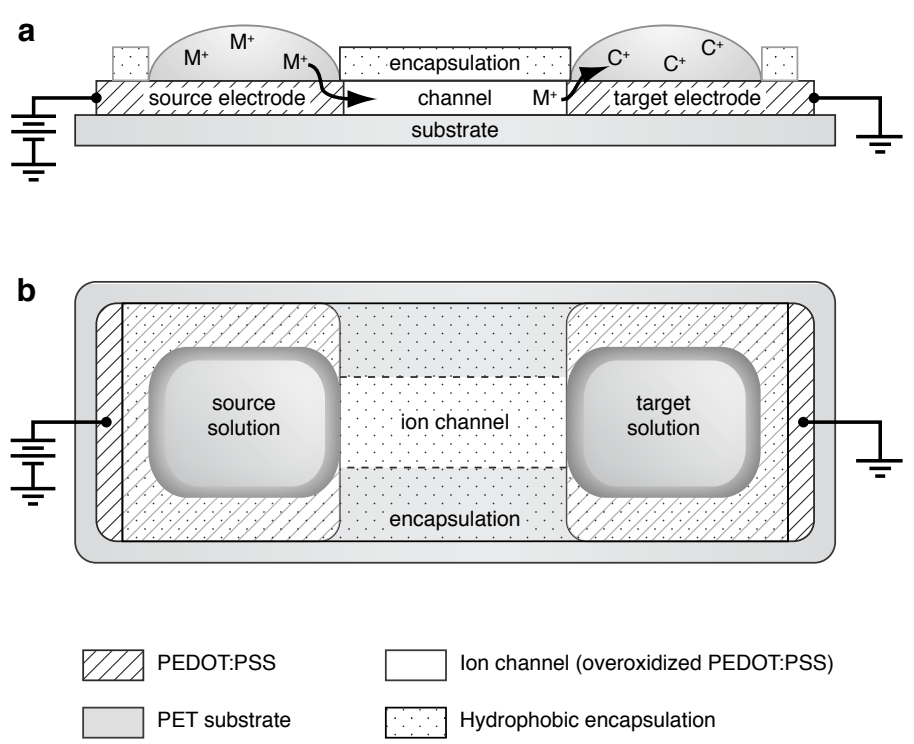

Figure 2: The organic electronic ion pump (OEIP). a, Side and, $\boldsymbol{b}$, top view of the device structure. $M^{+}$and $C^{+}$represent cations in source and target electrolyte, respectively, where $M^{+}$is the species "pumped" through the channel.

and in the absence of fluid flow - i.e., only molecules are delivered, not solution. We explore these devices to create precision microenvironments and to precisely regulate the cellular responses of several cell lines, such as $\mathrm{HCN}-2$ neuronal cells.

In addition to their now widespread utilization in LEDs and solar cells, conjugated polymers have been explored for more unique applications based on their electrochemical characteristics. The redox-activated swelling and contraction of certain polymers has led to the development of various actuator technologies [3], whereas the electrochemical modulation of electronic conductivity has paved the way for mixed electronic-ionic transistors [4,5], and light-emitting electrochemical cells (LECs) [6]. More recently, the organic electronics field has begun to merge with bio-medical research, yielding a host of biosensors, bio-electrodes, and drug delivery platforms $[7,8]$.

For delivery devices in particular, while many great strides in the technology have been achieved, there is still room for much improvement. These devices typically suffer from limitations such as low release rates and poor on/off ratios, as well as inadequate electronic control of the 
delivered dose [9]. Micro- and nanofluidic techniques have successfully bypassed some of these problems and have been used to generate controlled concentration gradients - analogous to many biological systems [10]. However, these systems are based on fluid flow, which necessarily disrupts the fragile liquid environment comprising the target system.

The OEIP reported here addresses these limitations, and can deliver a broad range of bio-substances including neurotransmitters - with precise electronic control and without convectional disturbances.

\section{THE OEIP DEVICE}

The OEIP consists of a single film of PEDOT:PSS photolithographically patterned into electrodes joined by an "ion channel" (Figure 2). The channel region, based on the same original film of PEDOT:PSS, is over-oxidized, thus deactivating electronic conduction while preserving ionic conduction [11]. A hydrophobic encapsulation layer, such as SU-8, covers the channel region, and provides openings over the electrodes for application of source and target electrolytes.

Upon application of a voltage between the electrodes, an electrochemical circuit is established, resulting in the oxidation of PEDOT at the anode and reduction of PEDOT at the cathode. Since the channel region is electronically insulating and the polyanionic PSS essentially forms a cation exchange membrane, the only mechanism whereby electronic current can be sustained is for cations from the anode/source side of the device be transported to the cathode/target side. Owing to the rapid ion exchange between the thin film PEDOT:PSS electrodes and their accompanying electrolytes, the source electrolyte serves as a reservoir for the positively charged species to be delivered, and the target electrolyte serves as the system into which species emerging out of the ion channel are immediately delivered (Figure 2).

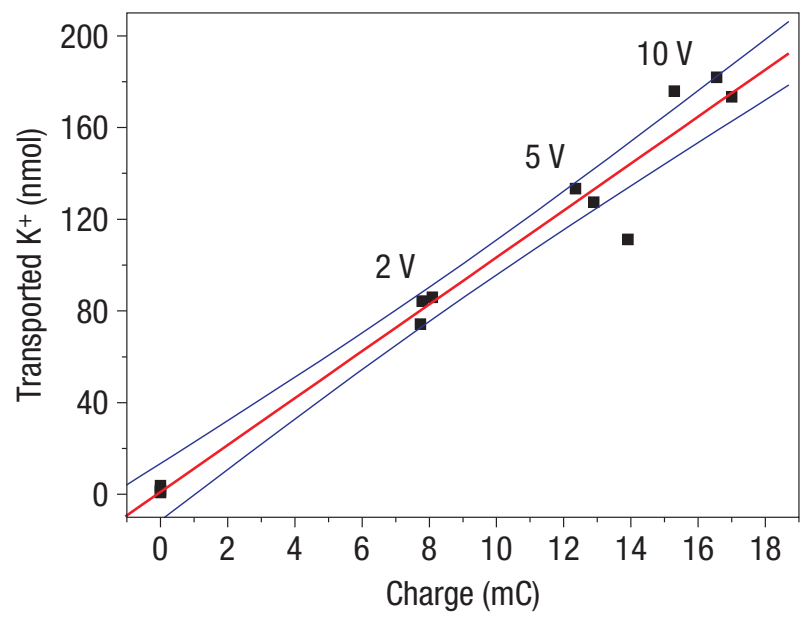

Figure 3: $\mathrm{K}^{+}$delivered to the target system versus charge measured in the electronic circuit (i.e., integrated current) at various driving voltages.
This electrophoretic delivery mechanism ensures that the delivery rate of positively charged species through the channel and into the target system is directly (and exactly) proportional to the current measured in the electronic branch of the circuit. Furthermore, since charged species, and not solution, are transported through the channel, material is delivered into the target system without convective flow. The OEIP therefore represents an ideal platform for precise, non-disruptive study of biological systems.

\section{RESULTS AND DISCUSSION Delivery Characteristics and Efficiency}

Using $\mathrm{KCl}$ as the source electrolyte, upon biasing the device, $\mathrm{K}^{+}$ions are transported through the channel and delivered into the target electrolyte. By comparing the amount of $\mathrm{K}^{+}$delivered into the target electrolyte (e.g., by atomic absorption spectroscopy) to the integrated current measured in the driving circuitry, a precise linear relationship can be established between the delivered concentration and the driving current (Figure 3). Indeed, these results indicate an approximately $100 \%$ efficiency, i.e., for every electron measured in the electronic circuit, exactly one $\mathrm{K}^{+}$ion is transported through the channel. In addition to $\mathrm{K}^{+}$, this precise electron-to-molecule ratio has been observed for all species successfully pumped to date. In addition, one or several miniaturized electrodes or ion channels can be incorporated to further refine the spatial resolution of the device.

\section{Using the OEIP to Induce pH Oscillations}

Many cell signaling events are defined by concentration oscillations. Here, we show that the OEIP device can be used to induce $\mathrm{pH}$ oscillations [12] in the target reservoir (Figure 4). When the voltage was applied $(5 \mathrm{~V}, 10 \mathrm{~s})$ protons were pumped out and the $\mathrm{pH}$ decreased rapidly close to the channel outlet. When the voltage was turned off the $\mathrm{pH}$ increased due to diffusion in the electrolyte. The diffusion process was slower than the delivery through pumping, and thereby limiting the frequency of which pulses can be applied to generate a regular $\mathrm{pH}$ oscillation.

\section{Pumping $\mathrm{K}^{+}$to Regulate the Intracellular $\mathrm{Ca}^{2+}$ Level in Neuronal Cells}

$\mathrm{K}^{+}$is an activator of excitable cells such as $\mathrm{HCN}-2$ neuronal cells. High extracellular $\left[\mathrm{K}^{+}\right]$depolarizes the plasma membrane, thus activating voltage-operated $\mathrm{Ca}^{2+}$ channels (VOCCs) in the membrane. The open channels allow a rapid influx of $\mathrm{Ca}^{2+}$ ions from the extracellular environment into the cytoplasm. We investigated whether $\mathrm{K}^{+}$pumping could activate the $\mathrm{Ca}^{2+}$ homeostasis in $\mathrm{HCN}-2$ cells. The cells were loaded with $\mathrm{Ca}^{2+}$ sensitive ratiometric probe FURA-2 AM and the intracellular $\mathrm{Ca}^{2+}$ level was recorded during the pumping experiment. 


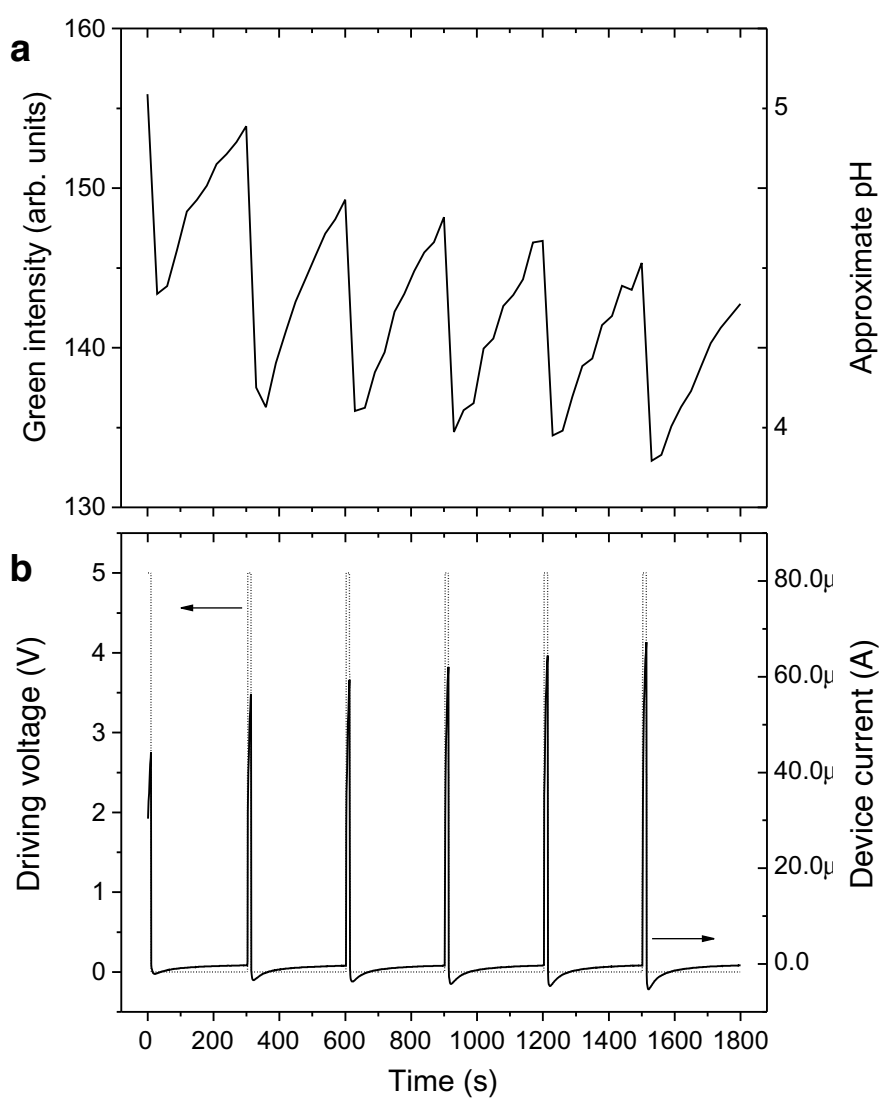

Figure 4: $p H$ modulation using the OEIP. a, The increase in $p H$ closest to the outlet of the ion channel into the target electrolyte. The $\mathrm{pH}$ was measured using a red $\mathrm{pH}$ indicator and analyzing the green intensity of subsequent microscope images. $\boldsymbol{b}$, The associated device current (solid) and voltage (dotted).

Figure 5 shows how the intracellular $\left[\mathrm{Ca}^{2+}\right]$ was modulated in $\mathrm{HCN}-2$ cells by pumping of $\mathrm{K}^{+}$. The dashed line shows how the intracellular $\left[\mathrm{Ca}^{2+}\right]$ increased after a few minutes of pumping. When the voltage-operated $\mathrm{Ca}^{2+}$ channels were blocked by $\mathrm{Gd}^{3+}$ (solid line) or nifedipine (dotted line) the intracellular $\left[\mathrm{Ca}^{2+}\right]$ remained unaffected thus proving the physiological relevance of the $\mathrm{Ca}^{2+}$ influx.

In cell signaling research, spatiotemporal control of ion fluxes is of great importance. To achieve local stimulation of cells the channel width was shrunk down to $50 \mu \mathrm{m}$. When $\mathrm{K}^{+}$was pumped with this device geometry, only cells close to the channel outlet responded while cells $500 \mu \mathrm{m}$ away were unresponsive.

\section{ONGOING WORK AND CONCLUSION}

Building on the results reported above, we are currently investigating further development of the OEIP platform. One focus is on the decrease of the device dimensions for even better spatial and temporal delivery resolution [13]. This will allow for true single-cell stimulation in vitro. In parallel, we are developing the device in an encapsulated scheme to enable its use in vivo as well as in expanded in vitro applications [14]. The

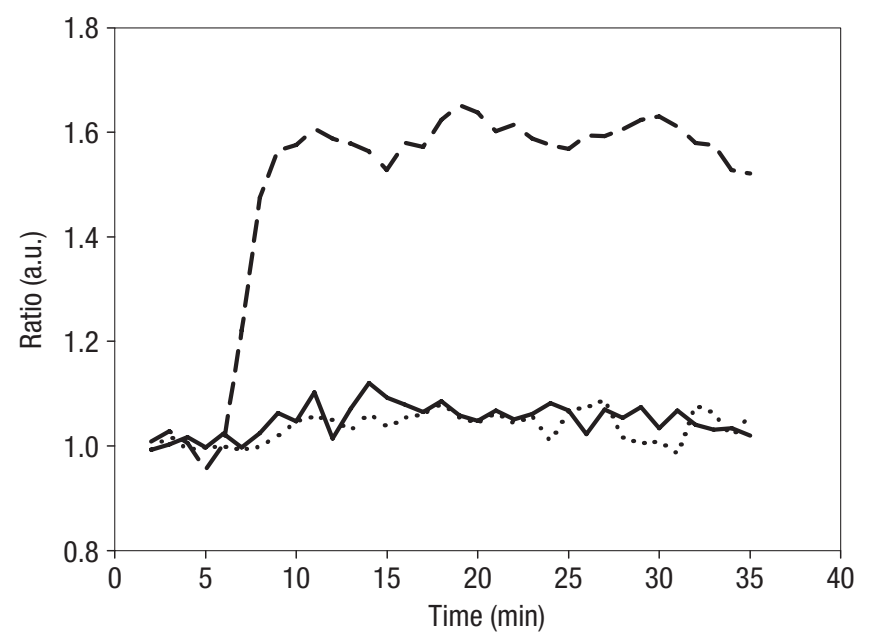

Figure 5: Fluorescence microscopy of intracellular $\mathrm{Ca}^{2+}$ levels in HCN-2 neuronal cells versus time. At 4 min, the pump was turned on, delivering $K^{+}$which resulted in (dashed line) increased response due to $\mathrm{K}^{+}$-opened $\mathrm{Ca}^{2+}$ channels, and (solid and dotted lines) no response when $\mathrm{Ca}^{2+}$ channels were blocked by secondary substances.

encapsulated form of the OEIP could lead toward fully implantable drug delivery devices. Finally, we are exploring new delivery profiles which could expand the stimulation capabilities of the OEIP, thus enabling better machine-to-biosystem interfacing.

The findings above, and our ongoing research efforts, promise for a versatile technology platform to precisely regulate physiology and signaling in vitro as well as in vivo.

\section{REFERENCES}

[1] L. Groenendaal, F. Jonas, D. Freitag, H. Pielartzik and J.R. Reynolds, "Poly(3,4ethylenedioxythiophene) and its derivatives: Past, present, and future", Advanced Materials, vol. 12, pp. 481-494, 2000.

[2] J. Isaksson, P. Kjäll, D. Nilsson, N. Robinson, M. Berggren and A. Richter-Dahlfors, "Electronic control of $\mathrm{ca}^{2+}$ signalling in neuronal cells using an organic electronic ion pump ", Nature Materials, vol. 6, pp. 673-679, 2007.

[3] E.W.H. Jager, E. Smela and O. Inganäs, "Microfabricating conjugated polymer actuators", Science, vol. 290, pp. 1540-1545, 2000.

[4] D. Nilsson, N. Robinson, M. Berggren and R. Forchheimer, "Electrochemical logic circuits", Advanced Materials, vol. 17, pp. 353-358, 2005.

[5] D.A. Bernards and G.G. Malliaras, "Steady-state and transient behavior of organic electrochemical transistors", Advanced Functional Materials, vol. 17, pp. 3538-3544, 2007. 
[6] Q. Pei, G. Yu, C. Zhang, Y. Yang and A.J. Heeger, "Polymer light-emitting electrochemical cells", Science, vol. 269, pp. 1086-1088, 1995.

[7] X. Cui, J. Wiler, M. Dzaman, R.A. Altschuler and D.C. Martin, "In vivo studies of polypyrrole/peptide coated neural probes", Biomaterials, vol. 24, pp. 777-787, 2003.

[8] N.K. Guimard, N. Gomez and C.E. Schmidt, "Conducting polymers in biomedical engineering", Progress in Polymer Science, vol. 32, pp. 876-921, 2007.

[9] P.M. George, D.A. Lavan, J.A. Burdick, C.-Y. Chen, E. Liang and R. Langer, "Electrically controlled drug delivery from biotin-doped conductive polypyrrole", Advanced Materials, vol. 18, pp. 577-581, 2006.

[10] N.L. Jeon, S.K.W. Dertinger, D.T. Chiu, I.S. Choi, A.D. Stroock and G.M. Whitesides, "Generation of solution and surface gradients using microfluidic systems", Langmuir, vol. 16, pp. 8311-8316, 2000.

[11] P. Tehrani, N.D. Robinson, T. Kugler, T. Remonen, L.-O. Hennerdal, J. Hall, A. Malmstrom, L. Leenders and M. Berggren, "Patterning polythiophene films using electrochemical over-oxidation", Smart Materials and Structures, vol. 14, pp. 21-25, 2005.
[12] J. Isaksson, P. Kjäll, D. Nilsson, N.D. Robinson, M. Berggren and A. Richter-Dahlfors, "Electronically controlled ph gradients and proton oscillations", Organic ELectronics, vol. 9, pp. 303-309, 2008.

[13] K. Tybrandt, K.C. Larsson, S. Kurup, D.T. Simon, P. Kjäll, J. Isaksson, M. Sandberg, E.W.H. Jager, A. Richter-Dahlfors and M. Berggren, "Translating electronic currents to precise acetylcholine-induced neuronal signaling using an organic electrophoretic delivery device", (submitted), 2009.

[14] D.T. Simon, S. Kurup, K.C. Larsson, R. Hori, K. Tybrandt, M. Goiny, E.W.H. Jager, M. Berggren, B. Canlon and A. Richter-Dahlfors, "Organic electronics for precise delivery of neurotransmitters to modulate mammalian sensory function", (submitted), 2009.

\section{CONTACT}

* E.W.H. Jager, tel: +46-11-363446; edwin.jager@itn.liu.se 